\title{
SWITCHNET: A NEURAL NETWORK MODEL FOR FORWARD AND INVERSE SCATTERING PROBLEMS
}

\author{
YUEHAW KHOO AND LEXING YING
}

\begin{abstract}
We propose a novel neural network architecture, SwitchNet, for solving the wave equation based inverse scattering problems via providing maps between the scatterers and the scattered field (and vice versa). The main difficulty of using a neural network for this problem is that a scatterer has a global impact on the scattered wave field, rendering typical convolutional neural network with local connections inapplicable. While it is possible to deal with such a problem using a fully connected network, the number of parameters grows quadratically with the size of the input and output data. By leveraging the inherent low-rank structure of the scattering problems and introducing a novel switching layer with sparse connections, the SwitchNet architecture uses much fewer parameters and facilitates the training process. Numerical experiments show promising accuracy in learning the forward and inverse maps between the scatterers and the scattered wave field.
\end{abstract}

\section{INTRODUCTION}

In this paper, we study the forward and inverse scattering problems via the use of artificial neural networks (NNs). In order to simplify the discussion, we focus on the time-harmonic acoustic scattering in two dimensional space. The inhomogeneous media scattering problem with a fixed frequency $\omega$ is modeled by the Helmholtz operator

$$
L u:=\left(-\Delta-\frac{\omega^{2}}{c^{2}(x)}\right) u,
$$

where $c(x)$ is the velocity field. In many settings, there exists a known background velocity field $c_{0}(x)$ such that $c(x)$ is identical to $c_{0}(x)$ except in a compact domain $\Omega$. By introducing the scatterer $\eta(x)$ compactly supported in $\Omega$

$$
\frac{\omega^{2}}{c(x)^{2}}=\frac{\omega^{2}}{c_{0}(x)^{2}}+\eta(x)
$$

we can equivalently work with $\eta(x)$ instead of $c(x)$. Note that in this definition $\eta(x)$ scales quadratically with the frequency $\omega$. However, as $\omega$ is assumed to be fixed throughout this paper, this scaling does not affect any discussion below.

In many real-world applications, $\eta(\cdot)$ is unknown. The task of the inverse problem is to recover $\eta(\cdot)$ based on some observation data $d(\cdot)$. The observation data $d(\cdot)$ is often a quantity derived from the Green's function $G=L^{-1}$ of the Helmholtz operator $L$ and, therefore, it depends closely on $\eta(\cdot)$. This paper is an exploratory attempt of constructing efficient approximations to the forward map $\eta \rightarrow d$ and the inverse map $d \rightarrow \eta$ using the modern tools from machine learning and artificial intelligence. Such approximations are highly useful for the numerical solutions of the scattering problems: an efficient map $\eta \rightarrow d$ provides an alternative to expensive partial differential equation (PDE) solvers for the Helmholtz equation; an efficient map $d \rightarrow \eta$ is more valuable as it allows us to solve the inverse problem of determining the scatterers from the scattering field, without going through the usual iterative process. 
In the last several years, deep neural network has become the go-to method in computer vision, image processing, speech recognition and many other machine learning applications [18, 28, 12, 9, More recently, methods based on NN have also been applied to solving PDEs. Based on the way that the NN is used, these methods for solving the PDE can be roughly separated into two different categories. For the methods in the first category [16, 27, 3, 10, 14, 6, instead of specifying the solution space via the choice of basis (as in finite element method or Fourier spectral method), NN is used for representing the solution. Then an optimization problem, for example an variational formulation, is solved in order to obtain the parameters of the NN and hence the solution to the PDE. Similar to the use of an NN for regression and classification purposes, the methods in the second category such as [23, 11, 13, 8, use an NN to learn a map that goes from the coefficients in the PDE to the solution of the PDE. As in machine learning, the architecture design of an NN for solving PDE usually requires the incorporation of the knowledge from the PDE domain such that the NN architecture is able to capture the behavior of the solution process. Despite the abundance of the works in using the NN for solving PDE, none of the above mentioned methods have tried to obtain the solution to the wave equation.

This paper takes a deep learning approach to learn both the forward and inverse maps. For the Helmholtz operator (1), we propose an NN architecture for determining the forward and inverse maps between the scatterer $\eta(\cdot)$ and the observation data $d(\cdot)$ generated from the scatterer. Although this task looks similar to the computer vision problems such as image segmentation, denoising, and super-resolution where the map between the two images has to be determined, the nature of the map in our problem is much more complicated. In many image processing tasks, the value of a pixel at the output generally only depends on a neighborhood of that pixel at the input layer. However, for the scattering problems, the input and output are often defined on different domains and, due to wave propagation, each location of the scatterer can influence every point of the scattered field. Therefore, the connectivity in the NN has to be wired in a non-local fashion, rendering typical NN with local connectivity insufficient. This leads to the development of the proposed SwitchNet. The key idea is the inclusion of a novel low-complexity switch layer that sends information between all pairs of sites effectively, following the ideas from butterfly factorizations 21. The same factorization was used earlier in the architecture proposed [19, but the network weights there are hardcoded and not trainable.

The paper is organized as followed. In Section 2, we discuss about some preliminary results concerning Helmholtz equation. In Section 3 , we study the so called far field pattern of the scattering problem, where the sources and receivers can be regarded as placed at infinity. We propose SwitchNet to determine the maps between the far field scattering pattern and the scatterer. In Section 4, we turn to the setting of a seismic imaging problem. In this problem, the sources and receivers are at a finite distance, but yet well-separated from the scatterer.

\section{Preliminary}

The discussion of this paper shall focus on the two-dimensional case. Here, we summarize the mathematical tools and notations used in this paper. As mentioned above, the scatterer $\eta(x)$ is compactly supported in a domain $\Omega$, whose diameter is of $O(1)$. For example, one can think of $\Omega$ to be the unit square centered at the origin. In (1), the Helmholtz operator is defined on the whole space $\mathbb{R}^{2}$ with the radiative (Sommerfeld) boundary condition [5] specified at infinity. Since the scatterer field $\eta(x)$ is localized in $\Omega$, it is convenient to truncate the computation domain to $\Omega$ by imposing the perfectly matched layer [1] that approximates the radiative boundary condition.

In a typical numerical solution of the Helmholtz operator, $\Omega$ is discretized by a Cartesian grid $X \subset \Omega$ at the rate of a few points per wavelength. As a result, the number of grid points $N$ per dimension is proportional to the frequency $\omega$. We simply use $\{x\}_{x \in X}$ to denote the discretization points of this 
$N \times N$ grid $X$. The Laplacian operator $-\Delta$ in the Helmholtz operator is typically discretized with local numerical schemes, such as the finite difference method [17. Via this discretization, we can consider the scatterer field $\eta$, discretized at the points in $X$, as a vector in $\mathbb{R}^{N^{2}}$ and the Helmholtz operator $L$ as a matrix in $\mathbb{C}^{N^{2} \times N^{2}}$.

Using the background velocity field $c_{0}(x)$, we first introduce the background Helmholtz operator $L_{0}=-\Delta-\omega^{2} / c_{0}^{2}$. With the help of $L_{0}$, one can write $L$ in a perturbative way as

$$
L=L_{0}-E, \quad E=\operatorname{diag}(\eta),
$$

where $E$ is viewed as a perturbation. By introducing the background Green's function

$$
G_{0}:=L_{0}^{-1},
$$

one can write down a formal expansion for the Green's function $G=L^{-1}$ of the $\eta$-dependent Helmholtz operator $L$ :

$$
\begin{aligned}
G & =\left(L_{0}\left(I-G_{0} E\right)\right)^{-1} \\
& \sim\left(I+G_{0} E+G_{0} E G_{0} E+\cdots\right) G_{0} \\
& \sim G_{0}+G_{0} E G_{0}+G_{0} E G_{0} E G_{0}+\cdots \\
& :=G_{0}+G_{1}+G_{2}+\cdots,
\end{aligned}
$$

which is valid when the scatterer field $\eta(x)$ is sufficiently small. The last line of the above equation serves as the definition of the successive terms of the expansion $\left(G_{1}, G_{2}\right.$, and so on). As $G_{0}$ can be computed from the knowledge of the background velocity field $c_{0}(x)$, most data gathering processes (with appropriate post-processing) focus on the difference $G-G_{0}=G_{1}+G_{2}+\cdots$ instead of $G$ itself.

A usual experimental setup consists of a set of sources $S$ and a set of receivers $R$ :

$$
S=\{s\}_{s \in S}, \quad R=\{r\}_{r \in R} .
$$

The data gathering process usually involves three steps: (1) impose an external force or incoming wave field via some sources, (2) solve for the scattering field either computationally or physically, (3) gather the data with receivers at specific locations or directions. The second step is modeled by the difference of the Green's function $G-G_{0}$, as we mentioned above. As for the other steps, it is convenient at this point to model the first step with a source-dependent operator $\Pi_{S}$ and the third one with a receiver-dependent operator $\Pi_{R}$. We shall see later how these operators are defined in more concrete settings. By putting these components together, one can set the observation data $d$ abstractly as

(6) $d=\Pi_{R}\left(G-G_{0}\right) \Pi_{S}=\Pi_{R}\left(G_{0} E G_{0}+G_{0} E G_{0} E G_{0}+\cdots\right) \Pi_{S}=\left(\Pi_{R} G_{0}\right)\left(E+E G_{0} E+\cdots\right)\left(G_{0} \Pi_{S}\right)$.

In this paper, we focus on two scenarios: far field pattern and seismic imaging. We start with far field pattern first to motivate and introduce SwitchNet. We then move on to the seismic case by focusing on the main differences.

\section{SWitchNet For FAR FIELD PATTERN}

3.1. Problem setup. In this section, we consider the problem of determining the map from the scatterer to the far field scattering pattern, along with its inverse map. Without loss of generality, we assume that the diameter of the domain $\Omega$ is of $O(1)$ after appropriate rescaling. The background velocity $c_{0}(x)$ is assumed to be 1 since the far field pattern experiments are mostly performed in free space.

In this problem, both the sources and the receivers are indexed by a set of unit directions in $\mathbb{S}^{1}$. The source associated with a unit direction $s \in S \subset \mathbb{S}^{1}$ is an incoming plane waves $e^{i \omega s \cdot x}$ pointing 
at direction $s$. It is well known that the scattered wave field, denoted by $u_{s}(x)$, at a large distance takes the following form [5]

$$
u_{s}(x)=\frac{e^{i \omega|x|}}{\sqrt{|x|}}\left(u_{s}^{\infty}\left(\frac{x}{|x|}\right)+o(1)\right),
$$

where the function $u_{s}^{\infty}(\cdot)$ is defined on the unit circle $\mathbb{S}^{1}$. The receiver at direction $r \in R \subset \mathbb{S}^{1}$ simply records the quantity $u_{s}^{\infty}(r)$ for each $s$. The set of observation data $d$ is then defined to be

$$
d(r s)=u_{s}^{\infty}(r) .
$$

Figure 1 provides an illustration of this experimental setup. Henceforth, we assume that both $R$ and $S$ are chosen to be a set of uniformly distributed directions on $\mathbb{S}^{1}$. Their size, denoted by $M$, typically scales linearly with frequency $\omega$.

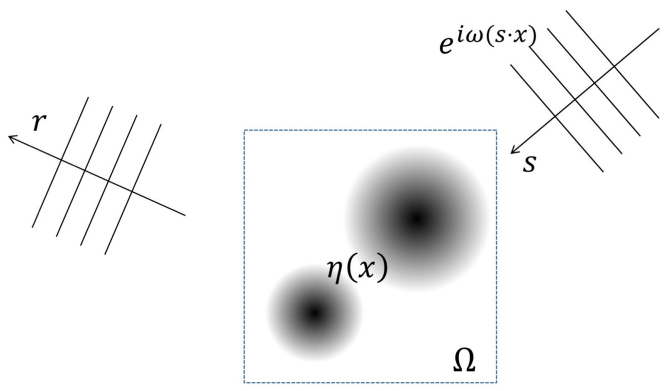

FIGURE 1 Illustration of the incoming and outgoing waves for a far field pattern problem. The scatterer $\eta(x)$ is compactly supported in the domain $\Omega$. The incoming plane wave points at direction $s$. The far field pattern is sampled at each receiver direction $r$.

This data gathering process can be put into the framework of (6). First, one can think of the source prescription as a limiting process that produces in the limit the incoming wave $e^{i \omega s \cdot x}$. The source can be considered to be located at the point $-s \rho$ for the direction $s \in \mathbb{S}^{1}$ with the distance $\rho \in \mathbb{R}^{+}$going to infinity. In order to compensate the geometric spreading of the wave field and also the phase shift, the source magnitude is assumed to scale like $\sqrt{\rho} e^{-i \omega \rho}$ as $\rho$ goes to infinity. Under this setup, we have

$$
\begin{aligned}
& \lim _{\rho \rightarrow \infty}\left(G_{0} \Pi_{S}\right)(x, s) \\
= & \lim _{\rho \rightarrow \infty}(1 / \sqrt{\rho}) e^{i \omega|x-(-s \rho)|} \sqrt{\rho} e^{-i \omega \rho} \\
= & \lim _{\rho \rightarrow \infty}(1 / \sqrt{\rho}) e^{i \omega(\rho+s \cdot x)} \sqrt{\rho} e^{-i \omega \rho} \\
= & e^{i \omega s \cdot x} .
\end{aligned}
$$

Similarly, one can also regard the receiver prescription as a limiting process as well. The receiver is located at point $r \rho^{\prime}$ for a fixed unit direction $r \in \mathbb{S}^{1}$ with $\rho^{\prime} \in \mathbb{R}^{+}$going to infinity. Again in order to to compensate the geometric spreading and the phase shift, one scales the received signal with $\sqrt{\rho^{\prime}} e^{-i \omega \rho^{\prime}}$. As a result, we have

$$
\begin{aligned}
& \lim _{\rho^{\prime} \rightarrow \infty}\left(\Pi_{R} G_{0}\right)(r, x) \\
= & \lim _{\rho^{\prime} \rightarrow \infty}\left(1 / \sqrt{\rho^{\prime}}\right) e^{i \omega\left|r \rho^{\prime}-x\right|} \sqrt{\rho^{\prime}} e^{-i \omega \rho^{\prime}}
\end{aligned}
$$




$$
\begin{aligned}
& =\lim _{\rho^{\prime} \rightarrow \infty}\left(1 / \sqrt{\rho^{\prime}}\right) e^{i \omega\left(\rho^{\prime}-r \cdot x\right)} \sqrt{\rho^{\prime}} e^{-i \omega \rho^{\prime}} \\
& =e^{-i \omega r \cdot x} .
\end{aligned}
$$

In this limiting setting, one redefine the observation data as

$$
d=\lim _{\rho, \rho^{\prime} \rightarrow \infty}\left(\Pi_{R} G_{0}\right)\left(E+E G_{0} E+\cdots\right)\left(G_{0} \Pi_{S}\right) .
$$

Now taking the two limits (7) and (8) under consideration, one arrives at the following representation of the observation data $d(r, s)$ for $r \in R$ and $s \in S$

$$
d(r, s)=\sum_{x \in X} \sum_{y \in X} e^{-i \omega r \cdot x}\left(E+E G_{0} E+\cdots\right)(x, y) e^{i \omega s \cdot y} .
$$

3.2. Low-rank property. The intuition behind the proposed NN architecture comes from examining (10) when $E$ (or $\eta$ ) is small. In such a situation, we simply retain the term that is linear in $E$. Using the fact that $E=\operatorname{diag}(\eta),(10)$ becomes

$$
d(r, s) \approx \sum_{x \in X} e^{i \omega(s-r) \cdot x} \eta(x)
$$

for $r \in R \subset \mathbb{S}^{1}$ and $s \in S \subset \mathbb{S}^{1}$. This linear map takes $\eta(x)$ defined on a Cartesian grid $X \subset \Omega$ to $d(r, s)$ defined on yet another Cartesian grid $R \times S \subset \mathbb{S}^{1} \times \mathbb{S}^{1}$. Recalling that both $R$ and $S$ are of size $M$ and working with a vectorized $d \in \mathbb{C}^{M^{2}}$, we can write the above equation compactly as

$$
d \approx A \eta
$$

where the element of the matrix $A \in \mathbb{C}^{M^{2} \times N^{2}}$ at $(r, s) \in R \times S$ and $x \in X$ is given by

$$
A(r s, x)=\exp (i \omega(s-r) \cdot x) \text {. }
$$

The following theorem concerning the matrix $A$ plays a key role in the design of our NN. Let us first partition $\Omega$ uniformly into $\sqrt{P_{X}} \times \sqrt{P_{X}}$ Cartesian squares of side-length equal to $1 / \sqrt{\omega}$. Here we assume that $\sqrt{P_{X}}$ is an integer. Note that, since the diameter of $\Omega$ is of $O(1), \sqrt{P_{X}} \approx \sqrt{\omega}$. This naturally partitions the set of grid points $X$ into $P_{X}$ subgroups depending on which square each point belongs to. We shall denote these subgroups by $X_{0}, \ldots, X_{P_{X}-1}$. Similarly, we also partition $\mathbb{S}^{1} \times \mathbb{S}^{1}$ uniformly (in the angular parameterization) into $\sqrt{P_{D}} \times \sqrt{P_{D}}$ squares $D_{0}, \ldots, D_{P_{D}-1}$ of side-length equal to $1 / \sqrt{\omega} \cdot \sqrt{P_{D}}$ is also assumed to be an integer and obviously $\sqrt{P_{D}} \approx \sqrt{\omega}$. This further partitions the set $R \times S$ into $P_{D}$ subgroups depending on which square they belong to. We shall denote these subgroups by $D_{0}, \ldots, D_{P_{D}-1}$. Fig. 2 illustrates the partition for $\sqrt{P_{X}}=\sqrt{P_{D}}=4$.
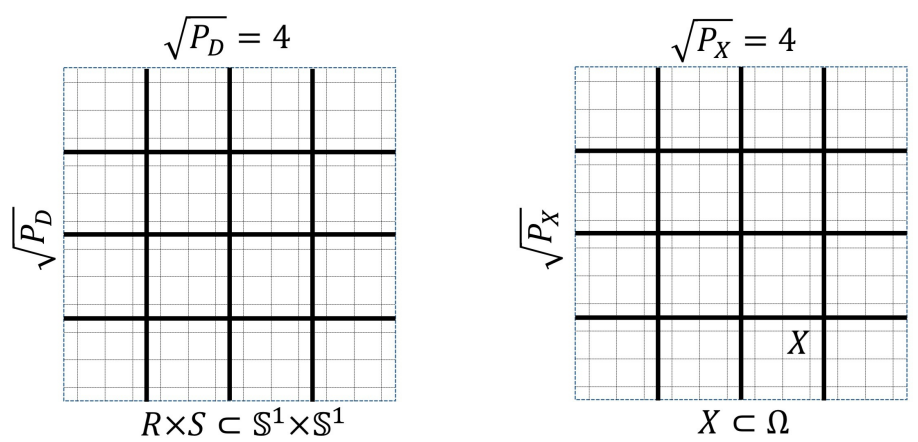

Figure 2 Illustration of the partitions used in Theorem 1. The fine grids stand for the Cartesian grids $X$ and $R \times S$. The bold lines are the boundary of the squares of the partitions. 
Theorem 1. For any $D_{i}$ and $X_{j}$, the submatrix

$$
A_{i j}:=[A(r s, x)]_{(r, s) \in D_{i}, x \in X_{j}}
$$

is numerically low-rank.

Proof. The proof of this theorem follows the same line of argument in 2, 29, 20, and below we outline the key idea. Denote the center of $D_{i}$ by $\left(r_{i}, s_{i}\right)$ and the center of $X_{j}$ by $x_{j}$. For each $(r, s) \in D_{i}$ and $x \in X_{j}$, we write

$$
\begin{aligned}
\exp (i \omega(s-r) \cdot x)=\exp \left(i \omega\left((s-r)-\left(s_{i}-r_{i}\right)\right) \cdot\left(x-x_{j}\right)\right) & \\
& \quad \exp \left(i \omega\left(s_{i}-r_{i}\right) \cdot x\right) \cdot \exp \left(i \omega(s-r) \cdot x_{j}\right) \cdot \exp \left(-i \omega\left(s_{i}-r_{i}\right) \cdot x_{j}\right) .
\end{aligned}
$$

Note that for fixed $D_{i}$ and $X_{j}$ each of the last three terms is either a constant or depends only on $x$ or $(r, s)$. As a result, $\exp (i \omega(s-r) \cdot x)$ is numerically low-rank if and only if the first term $\exp \left(i \omega\left((s-r)-\left(s_{i}-r_{i}\right)\right) \cdot\left(x-x_{j}\right)\right)$ is so. Such a low-rank property can be derived from the conditions concerning the side-lengths of $D_{i}$ and $X_{j}$. More precisely, since $(r, s)$ resides in $D_{i}$ with center $\left(r_{i}, s_{i}\right)$, then

$$
\left|(s-r)-\left(s_{i}-r_{i}\right)\right| \leq \frac{1}{\sqrt{\omega}} .
$$

Similarly as $x$ resides in $X_{j}$ with center $x_{j}$, then

$$
\left|x-x_{j}\right| \leq \frac{1}{\sqrt{\omega}}
$$

Multiplying these two estimates results in the estimate

$$
\left.\omega \mid\left((s-r)-\left(s_{i}-r_{i}\right)\right) \cdot\left(x-x_{j}\right)\right) \mid \leq 1
$$

for the phase of $\exp \left(i \omega\left((s-r)-\left(s_{i}-r_{i}\right)\right) \cdot\left(x-x_{j}\right)\right)$. Therefore,

$$
\exp \left(i \omega\left((s-r)-\left(s_{i}-r_{i}\right)\right) \cdot\left(x-x_{j}\right)\right)
$$

for $(r, s) \in D_{i}$ or $x \in X_{j}$ is non-oscillatory and hence can be approximated effectively by applying, for example, Chebyshev interpolation in both the $(r, s)$ and $x$ variables. Since the degree of the Chebyshev polynomials only increases poly-logarithmically with respect to the desired accuracy, $\exp \left(i \omega\left((s-r)-\left(s_{i}-r_{i}\right)\right) \cdot\left(x-x_{j}\right)\right)$ is numerically low-rank by construction. This proves that the submatrix $A_{i j}$ defined in $(13)$ is also numerically low-rank.

3.3. Matrix factorization. In this subsection, we show that Theorem 1 guarantees a low-complexity factorization of the matrix $A$. Let the row and column indices of $A \in \mathbb{C}^{M^{2} \times N^{2}}$ be partitioned into index sets $\left\{D_{i}\right\}_{i=0}^{P_{D}-1}$ and $\left\{X_{j}\right\}_{j=0}^{P_{X}-1}$, respectively, as in Theorem 1. To simplify the presentation, we assume $P_{X}=P_{D}=P,\left|X_{0}\right|=\cdots\left|X_{P-1}\right|=N^{2} / P$, and $\left|D_{0}\right|=\cdots=\left|D_{P-1}\right|=M^{2} / P$.

Since the submatrix

$$
A_{i j}:=[A(r s, x)]_{r s \in D_{i}, x \in X_{j}}
$$

is numerically low-rank, assume that

$$
A_{i j} \approx U_{i j} V_{i j}^{*}
$$

where $U_{i j} \in \mathbb{C}^{M^{2} / P \times t}$ and $V_{i j} \in \mathbb{C}^{N^{2} / P \times t}$. Here $t$ can be taken to be the maximum of the numerical ranks of all submatrices $A_{i j}$. Theorem 1 implies that $t$ is a small constant. 
By applying (19) to each block $A_{i j}, A$ can be approximated by

$$
\left[\begin{array}{cccc}
U_{00} V_{00}^{*} & U_{01} V_{01}^{*} & \cdots & U_{0(P-1)} V_{0(P-1)}^{*} \\
U_{10} V_{10}^{*} & U_{11} V_{11}^{*} & \cdots & U_{1(P-1)} V_{1(P-1)}^{*} \\
\vdots & & \ddots & \vdots \\
U_{(P-1) 0} V_{(P-1) 0}^{*} & U_{(P-1) 1} V_{(P-1) 1}^{*} & \cdots & U_{(P-1)(P-1)} V_{(P-1)(P-1)}^{*}
\end{array}\right] .
$$

The next step is to write 20 into a factorized form. First, introduce $U_{i}$ and $V_{j}$

$$
U_{i}=\left[U_{i 0}, U_{i 1}, \cdots, U_{i(P-1)}\right] \in \mathbb{C}^{M^{2} / P \times t P}, \quad V_{j}=\left[V_{0 j}, V_{1 j}, \cdots, V_{(P-1) j}\right] \in \mathbb{C}^{N^{2} / P \times t P},
$$

and define in addition

$$
U=\left[\begin{array}{llll}
U_{0} & & & \\
& U_{1} & & \\
& & \ddots & \\
& & & U_{P-1}
\end{array}\right] \in \mathbb{C}^{M^{2} \times P^{2} t}, \quad V^{*}=\left[\begin{array}{llll}
V_{0}{ }^{*} & & & \\
& V_{1}{ }^{*} & & \\
& & \ddots & \\
& & V_{P-1}{ }^{*}
\end{array}\right] \in \mathbb{C}^{P^{2} t \times N^{2},}
$$

In addition, introduce

$$
\Sigma=\left[\begin{array}{cccc}
\Sigma_{00} & \Sigma_{01} & \cdots & \Sigma_{0(P-1)} \\
\Sigma_{10} & \Sigma_{11} & \cdots & \Sigma_{1(P-1)} \\
\vdots & & \ddots & \vdots \\
\Sigma_{(P-1) 0} & \Sigma_{(P-1) 1} & \cdots & \Sigma_{(P-1)(P-1)}
\end{array}\right] \in \mathbb{C}^{P^{2} t \times P^{2} t}
$$

where the submatrix $\Sigma_{i j} \in \mathbb{C}^{P t \times P t}$ itself is a $P \times P$ block matrix with blocks of size $t \times t . \Sigma_{i j}$ is defined to be zero everywhere except being the identity matrix at the $(j, i)$-th $t \times t$ block. In order to help understand the NN architecture discussed below sections, it is imperative to understand the meaning of $\Sigma$. Let us assume for simplicity that $t=1$. Then for an arbitrary vector $z \in \mathbb{C}^{P^{2}}, \Sigma z$ essentially performs a "switch" that shuffles $z$ as follows

$$
(\Sigma z)(j P+i)=z(i P+j), \quad i, j=0, \ldots, P-1 .
$$

With the above definitions for $U, V$, and $\Sigma$, the approximation in 20 can be written compactly as

$$
A \approx U \Sigma V^{*} .
$$

Notice that although $A$ has $M^{2} \times N^{2}$ entries, using the factorization $(25), A$ can be stored using $t P\left(M^{2}+P+N^{2}\right)$ entries. In this paper, $P \approx \max (M, N)$ and $M$ and $N$ are typically on the same order. Therefore, instead of $O\left(N^{4}\right)$, one only needs $O\left(N^{3}\right)$ entries to parameterize the map $A$ approximately using (25). Such a factorization is also used in 21 for the compression of Fourier integral operators.

We would like to comment on another property that may lead to further reduction in the parameters used for approximating $A$. Let us focus on any two submatrices $A_{i j}$ and $A_{i k}$ of $A$. For two regions $X_{j}$ and $X_{k}$, where the center of $X_{j}$ and $X_{k}$ are $x_{j}$ and $x_{k}$ respectively, $X_{k}=X_{j}+\left(x_{k}-x_{j}\right)$. Let $(r, s) \in D_{i}$. For $x \in X_{j}$ and $x^{\prime}=x+\left(x_{k}-x_{j}\right) \in X_{k}$, we have

$$
\begin{aligned}
\exp (i \omega(s-r) \cdot x) & =g_{1}(r, s) h((r, s), x), \\
\exp \left(i \omega(s-r) \cdot x^{\prime}\right) & =g_{2}(r, s) h((r, s), x),
\end{aligned}
$$

where

$g_{1}(r, s)=\exp \left(i \omega(s-r) \cdot x_{j}\right), \quad g_{2}(r, s)=\exp \left(i \omega(s-r) \cdot x_{k}\right), \quad h((r, s), x)=\exp \left(i \omega(s-r) \cdot\left(x-x_{j}\right)\right)$. 
Therefore, the low-rank factorizations of $A_{i j}$ and $A_{i k}$ are solely determined by the factorization of $h(r s, x)$. This implies that it is possible to construct low-rank factorizations for $A_{i j}$ and $A_{i k}$ :

$$
A_{i j} \approx U_{i j} V_{i j}^{*}, \quad A_{i k} \approx U_{i k} V_{i k}^{*},
$$

such that $V_{i j}^{*}=V_{i k}^{*}$. Since this is true for all possible $j, k$, one can pick low-rank factorizations so that $V_{0}=V_{1}=\cdots=V_{P-1}$.

As a final remark in this section, this low complexity factorization 25 for $A$ can be easily converted to one for $A^{*}$ since

$$
A^{*} \approx V \Sigma^{*} U^{*}
$$

where $U, \Sigma, V$ are provided in $(21),(22)$, and $(23)$.

3.4. Neural networks. Based on the low-rank property of $A$ in Section 3.2 and its low-complexity factorization in Section 3.3 , we propose new NN architectures for representing the inverse map $d \rightarrow \eta$ and the forward map $\eta \rightarrow d$.

3.4.1. $N N$ for the inverse map $d \rightarrow \eta$. As pointed out earlier, $d \approx A \eta$ when $\eta$ is sufficiently small. The usual filtered back-projection algorithm [25] solves the inverse problem $d \rightarrow \eta$ via

$$
\eta \approx\left(A^{*} A+\epsilon I\right)^{-1} A^{*} d,
$$

where $\epsilon$ is the regularization parameter. In the far field pattern problem, $\left(A^{*} A+\epsilon I\right)^{-1}$ can be understood as a deconvolution operator. To see this, a direct calculation reveals that

$$
\left(A^{*} A\right)(x, y)=\sum_{r s \in R \times S} e^{i \omega(s-r) \cdot y} e^{-i \omega(s-r) x}=\sum_{r s \in R \times S} e^{-i \omega(s-r)(x-y)}
$$

for $x, y \in X$. (31) shows that $A^{*} A$ is a translation-invariant convolution operator. Therefore, the operator $\left(A^{*} A+\epsilon I\right)^{-1}$, as a regularized inverse of $A^{*} A$, simply performs a deconvolution. In summary, the above discussion shows that in order to obtain $\eta$ from the scattering pattern $d$ in the regime of small $\eta$, one simply needs to apply sequentially to $d$

- the operator $A^{*}$,

- a translation-invariant filter that performs the deconvolution $\left(A^{*} A+\epsilon I\right)^{-1}$.

Although these two steps might be sufficient when $\eta$ is small, a nonlinear solution is needed when $\eta$ is not so. For this purpose, we propose a nonlinear neural network SwitchNet for the inverse map. There are two key ingredients in the design of SwitchNet.

- The first key step is the inclusion of a Switch layer that sends local information globally, as depicted in Figure 4. The structure of the Switch layer is designed to mimic the matrix-vector multiplication of the operator $A^{*} \approx V \Sigma^{*} U^{*}$ in $(20)$. However unlike the fixed coefficients in (20), as an NN layer, the Switch layer allows for tunable coefficients and learns the right values for the coefficients from the training data. This gives the architecture a great deal of flexibility.

- The second key step is to replace the linear deconvolution in the back-projection algorithm with a few convolution (Conv) layers. This enriches the architecture with nonlinear capabilities when approximating the nonlinear inverse map.

The pseudo-code for SwitchNet is summarized in Algorithm 1 The input $d$ is a $\mathbb{C}^{M \times M}$ matrix, while the output $\eta$ is a $\mathbb{C}^{N \times N}$ matrix. The first three steps of Algorithm 1 mimics the application of the operator $A^{*} \approx V \Sigma^{*} U^{*}$. The Switch layer does most of the work, while the Vect and Square layers are simply operations that reshape the input and output data to the correct matrix form at the beginning and the end of the Switch layer. In particular, Vect groups the entries of the 2D field $d$ according to squares defined by the partition $D_{0}, \ldots, D_{P_{D}-1}$ and Square does the opposite. The 


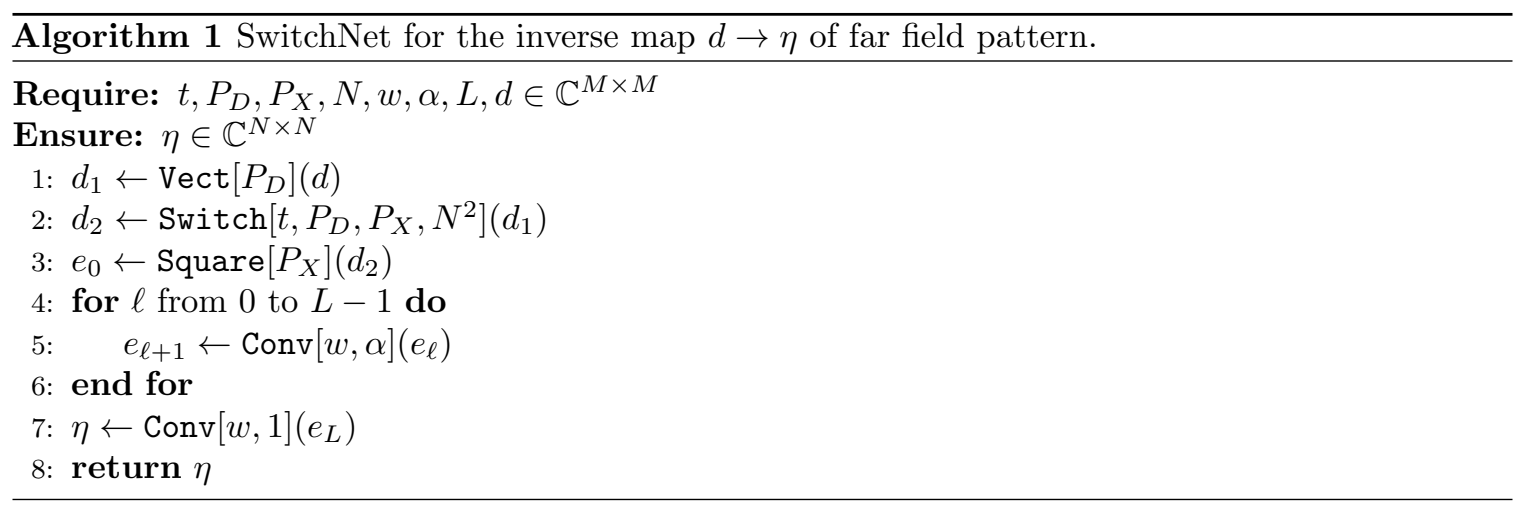

remaining lines of Algorithm 1 simply apply the Conv layers with window size $w$ and channel number $\alpha$.

These basic building blocks of SwitchNet are detailed in the following subsection. We also take the opportunity to include the details of the pointwise multiplication PM layer that will be used in later on.

3.4.2. Layers for SwitchNet. In this section we provide the details for the layers that are used in SwitchNet. Henceforth, we assume that the entries of a tensor is enumerated in the Python convention, i.e., going through the dimensions from the last one to the first. One operation that will be used often is a reshape, in which a tensor is changed to a different shape with the same number of entries and with the enumeration order of the entries kept unchanged.

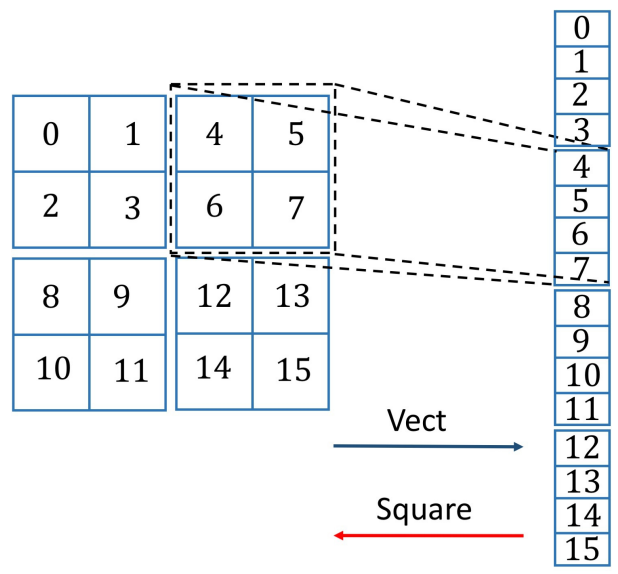

Figure 3 An illustration of the Vect and Square layers. The detail descriptions of the layers are provided in Section 3.4.2. For the purpose of illustration we let $P=4$. The Vect layer vectorize a $4 \times 4$ matrix on the left hand side according to the partitioning by $2 \times 2$ blocks, to give the size 16 vector on the right hand side. The Square layer is simply the adjoint map of the Vect layer.

Vectorize layer. $z_{\mathrm{O}}=\operatorname{Vect}[P]\left(z_{\mathrm{I}}\right)$ with input $z_{\mathrm{I}} \in \mathbb{C}^{n \times n}$. Henceforth we assume that $\sqrt{P}$ is an integer and $\sqrt{P}$ divides $n$. This operation partitions $z_{\mathrm{I}}$ into $\sqrt{P} \times \sqrt{P}$ square sub-blocks of equal size. Then each sub-block is vectorized, and the vectorized sub-blocks are stacked together as a vector in 
$\mathbb{C}^{n^{2}}$. Intuitively, these operations cluster the nearby entries in a sub-block together. The details of the Vect layer are given in the following:

- Reshape the $z_{\mathrm{I}}$ to a $\sqrt{P} \times \frac{n}{\sqrt{P}} \times \sqrt{P} \times \frac{n}{\sqrt{P}}$ tensor.

- Swap the second and the third dimensions to get a $\sqrt{P} \times \sqrt{P} \times \frac{n}{\sqrt{P}} \times \frac{n}{\sqrt{P}}$ tensor.

- Reshape the result to an $n^{2}$ vector and set it to $z_{\mathrm{O}}$.

Square layer. $z_{\mathrm{O}}=$ Square $[P]\left(z_{\mathrm{I}}\right)$ with input $z_{\mathrm{I}} \in \mathbb{C}^{n^{2}}$, where $\sqrt{P}$ is an integer. The output is $z_{\mathrm{O}} \in \mathbb{R}^{n \times n}$. Essentially as the adjoint operator of the Vect layer, this layer fills up each square sub-block of the matrix $z_{\mathrm{O}}$ with a segment of entries in $z_{\mathrm{I}}$. The details are given as followed:

- Reshape the $z_{\mathrm{I}}$ to a $\sqrt{P} \times \sqrt{P} \times \frac{n}{\sqrt{P}} \times \frac{n}{\sqrt{P}}$ tensor.

- Swap the second and the third dimensions to get a $\sqrt{P} \times \frac{n}{\sqrt{P}} \times \sqrt{P} \times \frac{n}{\sqrt{P}}$ tensor.

- Reshape the result to an $n \times n$ matrix and set it to $z_{\mathrm{O}}$.

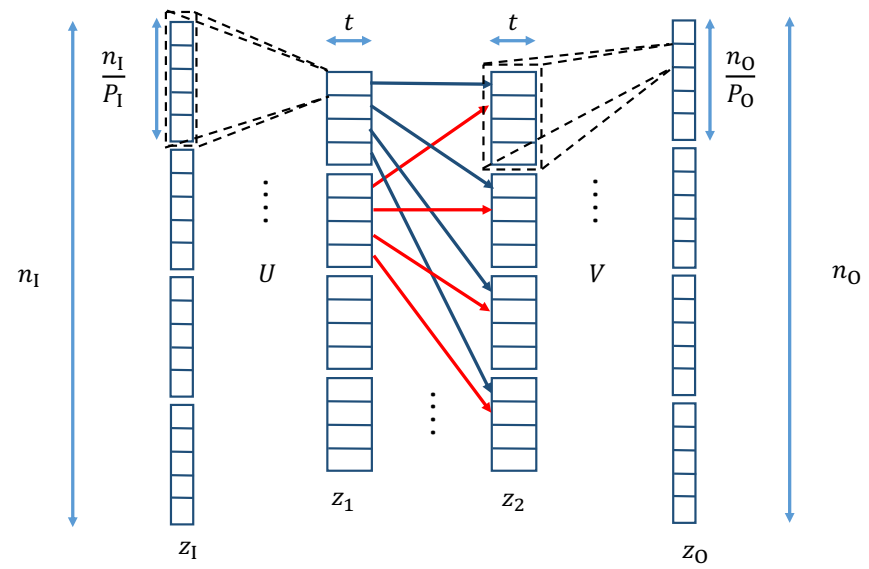

FiguRE 4 An illustration of the Switch layer where the detail description of it is provided in Section 3.4.2. For the purpose of illustration we let $n_{\mathrm{I}}=n_{\mathrm{O}}=20, P_{\mathrm{I}}=$ $P_{\mathrm{O}}=4$.

Switch layer. $z_{\mathrm{O}}=\operatorname{Switch}\left[t, P_{\mathrm{I}}, P_{\mathrm{O}}, n_{\mathrm{O}}\right]\left(z_{\mathrm{I}}\right)$ with input $z_{\mathrm{I}} \in \mathbb{C}^{n_{\mathrm{I}}}$. It is assumed that $n_{\mathrm{I}}$ and $n_{\mathrm{O}}$ are integer multiples of $P_{\mathrm{I}}$ and $P_{\mathrm{O}}$, respectively. This layer consists the following steps.

- Apply $U^{T}$ to $z_{\mathrm{I}}$ :

$$
\begin{aligned}
& z_{1}=U^{T} z_{\mathrm{I}} \in \mathbb{C}^{P_{\mathrm{O}} P_{\mathrm{I}} t}, \\
& U^{T}=\left[\begin{array}{ccc}
U_{0}^{T} & & \\
& \ddots & \\
& & U_{P_{\mathrm{I}}-1}^{T}
\end{array}\right], \quad U_{0}^{T}, \ldots, U_{P_{\mathrm{I}}-1}^{T} \in \mathbb{C}^{t P_{\mathrm{O}} \times \frac{n_{\mathrm{I}}}{P_{\mathrm{I}}}}
\end{aligned}
$$

- Reshape $z_{1}$ to be a $\mathbb{C}^{P_{\mathrm{O}} \times P_{\mathrm{I}} \times t}$ tensor. Here we follow the Python convention of going through the dimensions from the last one to the first one. Then a permutation is applied to swap the first two dimensions to obtain a tensor of size $\mathbb{C}^{P_{\mathrm{O}} \times P_{\mathrm{I}} \times t}$. Finally, the result is reshaped to a vector $z_{2} \in \mathbb{C}^{P_{\mathrm{I}} P_{\mathrm{O}} t}$ again going through the dimensions from the last to the first. 
- Apply $V$ to $z_{2}$ :

$$
\begin{gathered}
z_{\mathrm{O}}=V z_{2} \in \mathbb{R}^{n_{\mathrm{O}}}, \\
V=\left[\begin{array}{ccc}
V_{0} & & \\
& \ddots & \\
& & V_{P_{\mathrm{O}}-1}
\end{array}\right], \quad V_{0}, \ldots, V_{P_{\mathrm{O}}-1} \in \mathbb{C}^{\frac{n_{\mathrm{O}}}{P_{\mathrm{O}}} \times t P_{\mathrm{I}}} .
\end{gathered}
$$

Here the non-zero entries of $U, V$ are the trainable parameters. The Switch layer is illustrated in Figure 4

Convolution layer. $z_{\mathrm{O}}=\operatorname{Conv}\left[w, c_{\mathrm{O}}\right]\left(z_{\mathrm{I}}\right)$ with input $z_{\mathrm{I}}=\mathbb{C}^{n \times n \times c_{\mathrm{I}}}$. Here $c_{\mathrm{I}}, c_{\mathrm{O}}$ denote the input and output channel numbers and $w$ denotes the window size. In this paper we only use the convolution layer with stride 1 and with zero-padding:

$$
\begin{aligned}
& z_{\mathrm{O}}\left(k_{1}, k_{2}, k_{3}\right)=\operatorname{ReLU}\left(\sum_{l_{1}=\max \left(0, k_{1}-\frac{w-1}{2}\right)}^{\min \left(n-1, k_{1}+\frac{w-1}{2}\right)} \sum_{l_{2}=\max \left(0, k_{2}-\frac{w-1}{2}\right)}^{\min \left(n-1, k_{2}+\frac{w-1}{2}\right)} \sum_{l_{3}=0}^{c_{\mathrm{I}}-1}\right. \\
&\left.W\left(l_{1}-k_{1}+\frac{w-1}{2}, l_{2}-k_{2}+\frac{w-1}{2}, l_{3}, k_{3}\right) z_{\mathrm{I}}\left(l_{1}, l_{2}, l_{3}\right)+b\left(k_{3}\right)\right)
\end{aligned}
$$

with $k_{1}, k_{2}=0, \ldots, n-1, k_{3}=0, \ldots, c_{\mathrm{O}}-1$. Here $\operatorname{ReLU}(x)=\max (0, x)$ and $w$ is assumed to be odd in the presentation. Both $W \in \mathbb{C}^{w \times w \times c_{\mathrm{I}} \times c_{\mathrm{O}}}$ and $b \in \mathbb{C}^{c_{\mathrm{O}}}$ are the trainable parameters.

Pointwise multiplication layer. $z_{\mathrm{O}}=\operatorname{PM}\left(z_{\mathrm{I}}\right)$ with input $z_{\mathrm{I}} \in \mathbb{C}^{n \times n \times c_{\mathrm{I}}}$. It is defined as

$$
z_{\mathrm{O}}\left(k_{1}, k_{2}\right)=W\left(k_{1}, k_{2}\right) z_{\mathrm{I}}\left(k_{1}, k_{2}\right)+b\left(k_{1}, k_{2}\right),
$$

$k_{1}, k_{2}=0, \ldots, n-1$. Both $W \in \mathbb{C}^{n \times n}$ and $b \in \mathbb{C}^{n \times n}$ are trainable parameters.

We remark that, among these layers, the Switch layer has the most parameters. If the input and output to the Switch layer both have size $n \times n$, the number of parameter is $2 t P n^{2}$ where $P$ is the number of squares that partition the input field and $t$ is the rank of the low-rank approximation.

3.4.3. $N N$ for the forward map $\eta \rightarrow d$. We move on to discuss the parameterization of the forward map $\eta \rightarrow d$. The proposal is based on the simple observation that the inverse of the inverse map is the forward map.

More precisely, we simply reverse the architecture of the inverse map proposed in Algorithm 1 This results in an NN presented Algorithm 2, The basic architecture of this NN involves applying a few layers of Conv first, then followed by a Switch layer that mimics $A \approx U \Sigma V^{*}$.

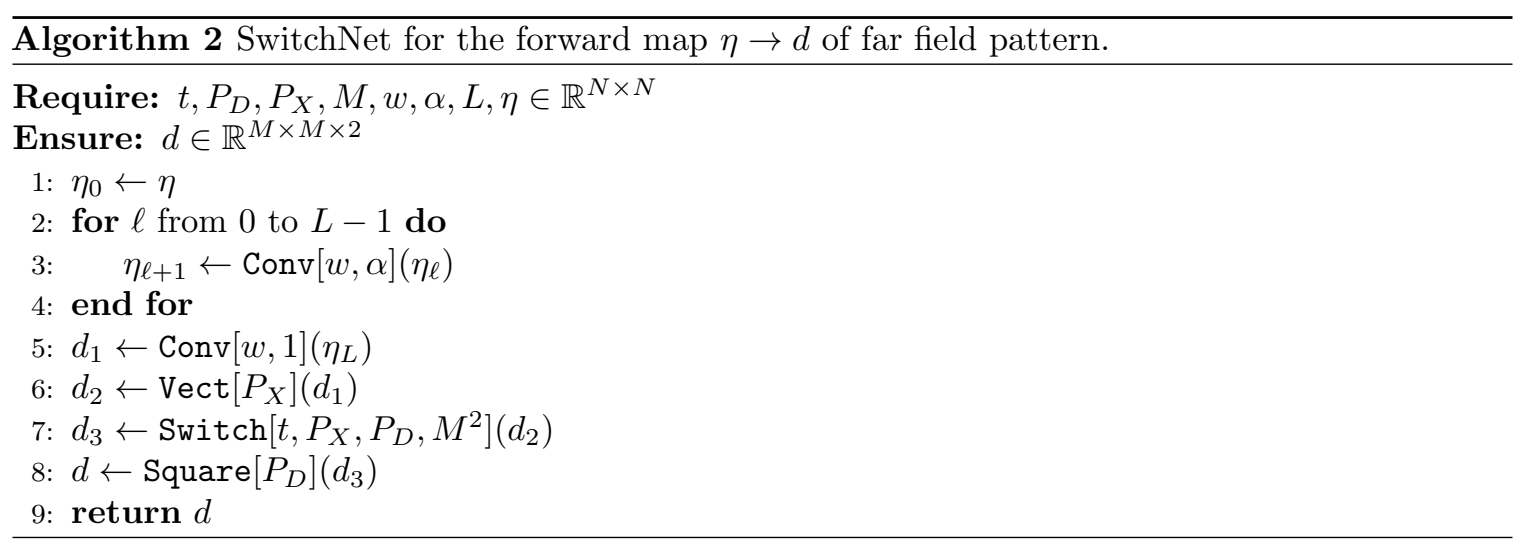


We would also like to mention yet another possibility to parameterize the forward map $\eta \rightarrow d$, via a recurrent neural network [24]. Let

$$
E_{\text {eff }}=E+E G_{0} E+E G_{0} E G_{0} E+\cdots=: E_{1}+E_{2}+E_{3}+\cdots .
$$

One can leverage the following recursion

$$
E_{k+1}=E G_{0} E_{k} \quad k=1,2, \ldots K
$$

to approximate $E_{\text {eff }}$ by treating each $E_{k}$ as an $N^{2} \times N^{2}$ image and using a recurrent neural network. At the $k$-th level of the recurrent neural network, it takes $E_{k}$ and $E$ as inputs and outputs $E_{k+1}$. More specifically, in order to go from $E_{k}$ to $E_{k+1}$, one first apply $G_{0}$ to each column of the image $E_{k}$, then each row of the image is reweighted by the diagonal matrix $E$. Stopping at the $K$-th level for a sufficiently large $K, E_{\text {eff }}$ can be approximated by

$$
E_{\mathrm{eff}} \approx \sum_{i=1}^{K+1} E_{i}
$$

Once holding such an approximation to $E_{\text {eff }}$, we plug it into 10

$$
d(r, s)=\sum_{x \in X} \sum_{y \in X} e^{i \omega r \cdot x} E_{\text {eff }}(x, y) e^{-i \omega s \cdot y}=\sum_{x \in X} e^{i \omega r \cdot x}\left(\sum_{y \in X} E_{\text {eff }}(x, y) e^{-i \omega s \cdot y}\right) .
$$

This shows that the map from $E_{\text {eff }}$ to $d$ can be realized by applying a matrix product to $E_{\text {eff }}$ first on the $y$-dimension, then on the $x$-dimension. If we view applying the Green's function $G_{0}$ as applying a convolution layer in an NN, the above discussion shows that the forward map can be obtained by first applying a recurrent NN followed by a convolutional NN. The main drawback of this approach is the large memory requirement (i.e., $N^{2} \times N^{2}$ ) to store each individual $E_{k}$. In addition, the use of a recurrent NN may lead to difficulty in training [26] due to the issue of exploding or vanishing gradient. Moreover, since the weights for parameterizing $G_{0}$ are shared over multiple layers in the recurrent $\mathrm{NN}$, one might not be able to efficiently use back-propagation, which may lead to a longer training time. These are the main reasons why we decided to adopt the approach in Algorithm 2.

3.5. Numerical results. In this section, we present numerical results of SwitchNet for far field pattern at a frequency $\omega \approx 60$. The scatterer field $\eta(x)$ supported in $\Omega=[-0.5,0.5]^{2}$ is assumed to be a mixture of Gaussians

$$
\sum_{i=1}^{n_{s}} \beta \exp \left(-\frac{\left|x-c_{i}\right|^{2}}{2 \sigma^{2}}\right)
$$

where $\beta=0.2$ and $\sigma=0.015$. When preparing the training and testing examples, the centers $\left\{c_{i}\right\}_{i=1}^{n_{s}}$ of the Gaussians are chosen to be uniformly distributed within $\Omega$. The number $n_{s}$ of the Gaussians in the mixture is set to vary between 2 and 4 . In the numerical experiments, the domain $\Omega=[-0.5,0.5]^{2}$ is discretized by an $80 \times 80$ Cartesian grid $X$. To discretize the source and receiver directions, we set both $R$ and $S$ to be a set of 80 equally spaced unit directions on $\mathbb{S}^{1}$. Therefore in this example, $N=M=80$.

In Algorithm 1, the parameters are specified as $t=3$ (rank of the low-rank approximation), $P_{X}=8^{2}, P_{D}=4^{2}, w=10$ (window size of the convolution layers), $\alpha=18$ (channel number of the convolution layers), and $L=3$ (number of convolution layers), resulting $3100 \mathrm{~K}$ number of parameters. The parameters for Algorithm 2 are chosen to be $t=4, P_{X}=8^{2}, P_{D}=4^{2}, w=10, \alpha=24$, and $L=3$, with a total of $4200 \mathrm{~K}$ parameters. Note that for both algorithms the number of parameters is significantly less than the one of a fully connected NN, which has at least $80^{4}=40960 \mathrm{~K}$ parameters.

SwitchNet is trained with the ADAM optimizer [15] in Keras [4] with a step size of 0.002 and a mini-batch size of size 200. The optimization is run for 2500 epochs. Both the training and testing 
data sets are obtained by numerically solving the forward scattering problem with an accurate finite difference scheme with a perfectly matched layer. In the experiment, $12.5 \mathrm{~K}$ pairs of $(\eta, d)$ are used for training, and another $12.5 \mathrm{~K}$ pairs are reserved for testing. The errors are reported using the mean relative errors

$$
\frac{1}{N_{\text {test }}} \sum_{i=1}^{N_{\text {test }}} \frac{\left\|d_{i}^{\mathrm{NN}}-d_{i}\right\|_{F}}{\left\|d_{i}\right\|_{F}}, \quad \frac{1}{N_{\text {test }}} \sum_{i=1}^{N_{\text {test }}} \frac{\left\|\eta_{i}^{\mathrm{NN}}-\eta_{i}\right\|_{F}}{\left\|\eta_{i}\right\|_{F}},
$$

where $d_{i}^{\mathrm{NN}}$ and $d_{i}$ denote the predicted and ground truth scattering patterns respectively for the $i$-th testing sample, and $\eta_{i}^{\mathrm{NN}}$ and $\eta_{i}$ denote the predicted and ground truth scatterer field respectively. Here $\|\cdot\|_{F}$ is the Frobenius norm.

Table 1 summarizes the test errors for Gaussian mixtures with different choices of $n_{s}$. For the purpose of illustration, we show the predicted $d$ and $\eta$ by SwitchNet along with the ground truth in Figure 5 for one typical test sample.

\begin{tabular}{ccc}
\hline \hline$n_{s}$ & Forward map & Inverse map \\
\hline \hline 2 & $9.4 \mathrm{e}-03$ & $1.2 \mathrm{e}-02$ \\
3 & $4.0 \mathrm{e}-02$ & $1.4 \mathrm{e}-02$ \\
4 & $4.8 \mathrm{e}-02$ & $2.4 \mathrm{e}-02$ \\
\hline
\end{tabular}

TABLE 1 Prediction error of SwitchNet for the maps $\eta \rightarrow d$ and $d \rightarrow \eta$ for far field pattern.

\section{SwitchNet For SeISmic IMAGing}

4.1. Problem setup. This section considers a two-dimensional model problem for seismic imaging. The scatterer $\eta(x)$ is again assumed to be supported in a domain $\Omega$ with an $O(1)$ diameter, after appropriate rescaling. $\Omega$ is discretized with a Cartesian grid $X=\{x\}_{x \in X}$ at the rate of at least a few point per wavelength. Compared to the source and receiver configurations in Section 3.1, the experiment setup here is simpler. One can regard both $S=\{s\}_{s \in S}$ and $R=\{r\}_{r \in R}$ to be equal to a set of uniformly sampled points along a horizontal line near the top surface of the domain. The support of $\eta$ is at a certain distance below the top surface so that it is well-separated from the sources and the receivers (see Figure 6 for an illustration of this configuration).

The source and receiver operators in (6) take a particularly simple form. For the sources, the operator $\left(G_{0} \Pi_{S}\right)$ is simply given by sampling:

$$
\left(G_{0} \Pi_{S}\right)(x, s)=G_{0}(x, s)
$$

Similarly for the receivers, the operator $\left(\Pi_{R}^{T} G_{0}\right)$ is given by

$$
\left(\Pi_{R} G_{0}\right)(r, y)=G_{0}(r, y) .
$$

After plugging these two formulas back into (6), one arrives at the following representation of the observation data $d(r, s)$ for $r \in R$ and $s \in S$

$$
d(r, s)=\sum_{x \in X} \sum_{y \in X} G_{0}(r, x)\left(E+E G_{0} E+\cdots\right)(x, y) G_{0}(y, s) .
$$




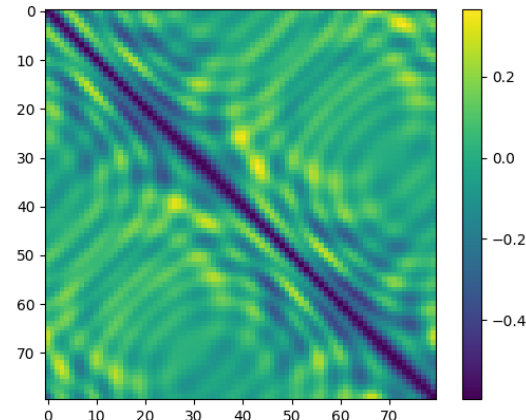

(A) Ground truth scattering pattern $d$.

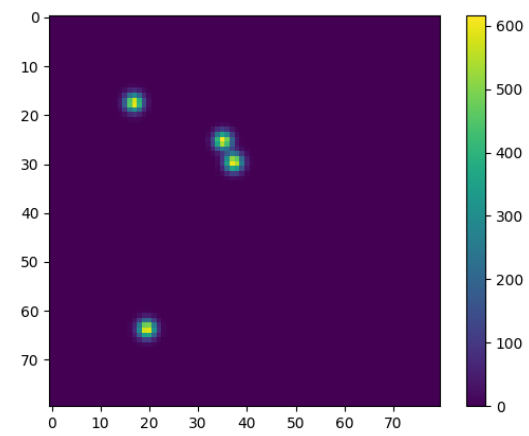

(c) Ground truth scatterers $\eta=$ $\omega^{2}\left(1 / c^{2}-1 / c_{0}^{2}\right)$.

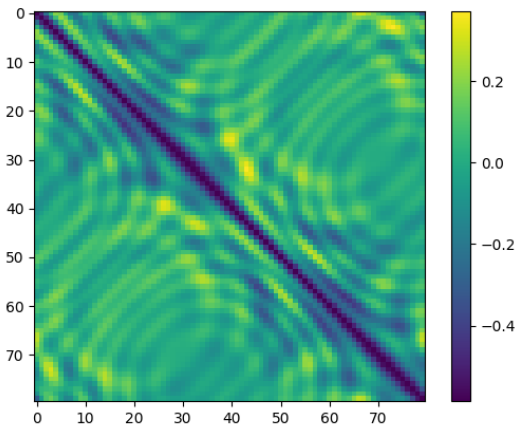

(B) Predicted scattering pattern $d^{\mathrm{NN}}$.

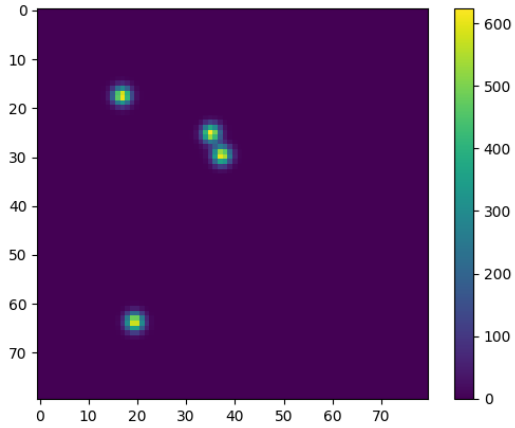

(D) Predicted scatterers $\eta^{\mathrm{NN}}$

FiguRE 5 Results for a typical instance of the far field pattern problem with $n_{s}=4$. (a) The ground truth scattering pattern. (b) The scattering pattern predicted by SwitchNet with a $4.9 \mathrm{e}-02$ relative error. (c) The ground truth scatterers. (d) The scatterers predicted by SwitchNet with a $2.4 \mathrm{e}-02$ relative error.

4.2. Low-rank property. Following the approach taken in Section 3.2, we start with the linear approximation under the assumption that $\eta(x)$ is weak. Since $E=\operatorname{diag}(\eta)$, the first order approximation is

$$
d(r, s) \approx \sum_{x \in X} G_{0}(r, x) G_{0}(x, s) \eta(x)
$$

By regarding $\eta$ as a vector in $\mathbb{R}^{N^{2}}$ and $d$ as a vector $\in \mathbb{C}^{M^{2}}$, one obtains the linear system

$$
d \approx A \eta, \quad A \in \mathbb{C}^{M^{2} \times N^{2}},
$$

where the element $A$ at $(r, s) \in R \times S$ and $x \in X$ is given by

$$
A(r s, x)=G_{0}(r, x) G_{0}(x, s)=G_{0}(r, x) G_{0}(s, x) .
$$




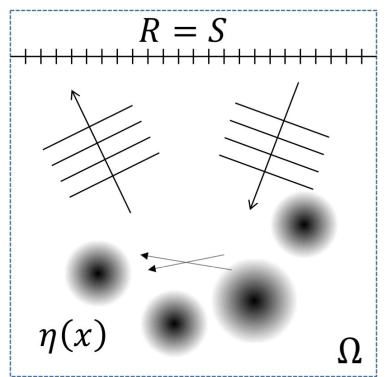

FiguRE 6 Illustration of a simple seismic imaging setting. The sources $(S)$ and receivers $(R)$ are located near the surface level (top) of the domain $\Omega$. The scatterer field $\eta(x)$ is assumed to be well-separated from the sources and the receivers.

Under the assumptions that the sources $S$ and receivers $R$ are well-separated from the support of $\eta(x)$ and that $c_{0}(x)$ varies smoothly, the matrix $A$ satisfies a low-rank property similar to Theorem 1. To see this, we again partition $X$ into Cartesian squares $X_{0}, \ldots, X_{P_{X}-1}$ of side-length equal to $1 / \sqrt{\omega}$. Since $R=S$ is now the restriction of $X$ on the surface level, this partition also induces a partitioning for $R \times S$. When $c_{0}(x)$ varies smoothly, it is shown (see for example [7]) that the restriction of the matrix $\left[G_{0}(r, x)\right]_{r \in R, x \in X}\left(\right.$ or $\left.\left[G_{0}(s, x)\right]_{s \in S, x \in X}\right)$ to each piece of the partitioning is numerically low-rank. Since the matrix $A$ is obtained by taking the Khatri-Rao product [22] of $\left[G_{0}(r, x)\right]_{r \in R, x \in X},\left[G_{0}(s, x)\right]_{s \in S, x \in X}$, the low-rank property is preserved with the guarantee that the rank at most squares in the worst case.

By following the same argument in Section 3.3 , one can show that the matrix $A$ has a lowcomplexity matrix factorization $A \approx U \Sigma V^{*}$ of exactly the same structure as $(20)$. The corresponding factorization for $A^{*}$ is $A^{*} \approx V \Sigma^{*} U^{*}$.

4.3. Neural networks. Based on the low-rank property in Section 4.2, we propose here SwitchNet for seismic imaging.

4.3.1. $N N$ for the inverse map $d \rightarrow \eta$. When the linear approximation is valid (i.e., (41) holds) $\eta$ can be obtained from $d$ via a filtered projection approach (or called migration in the seismic community)

$$
\eta \approx\left(A^{*} A+\epsilon I\right)^{-1} A^{*} d,
$$

where $\epsilon I$ is a regularizing term. Since $A^{*}$ has a low-complexity factorization $A^{*} \approx V \Sigma^{*} U^{*}$, the application $A^{*}$ to a vector can be represented by a Switch layer.

Concerning the $\left(A^{*} A+\epsilon I\right)^{-1}$ term, note that

$$
\left(A^{*} A\right)(x, y) \approx \sum_{r s \in R \times S} \overline{G_{0}(x, r) G_{0}(x, s)} G_{0}(r, y) G_{0}(s, y),
$$

which, unlike (31), is no longer a translation-invariant kernel as the data gathering setup is not so. For example, even when the background velocity $c_{0}(x)=1$, the different terms of the Green's function $G_{0}(\cdot)$ in $(44)$ scale like

$$
\frac{1}{\sqrt{|x-r|}}, \quad \frac{1}{\sqrt{|x-s|}}, \quad \frac{1}{\sqrt{|y-r|}}, \quad \frac{1}{\sqrt{|x-s|}},
$$

which fail to give a translation-invariant kernel of form $K(x-y)$. As a direct consequence, the operator $\left(A^{*} A+\epsilon I\right)^{-1}$ is not translation-invariant either. 
In order to capture the loss of translation-invariance, we include an extra pointwise multiplication layer PM (defined in Section 3.4.2 when dealing with the inverse map. The pseudo-code of the NN for the inverse map is given in Algorithm 3 .

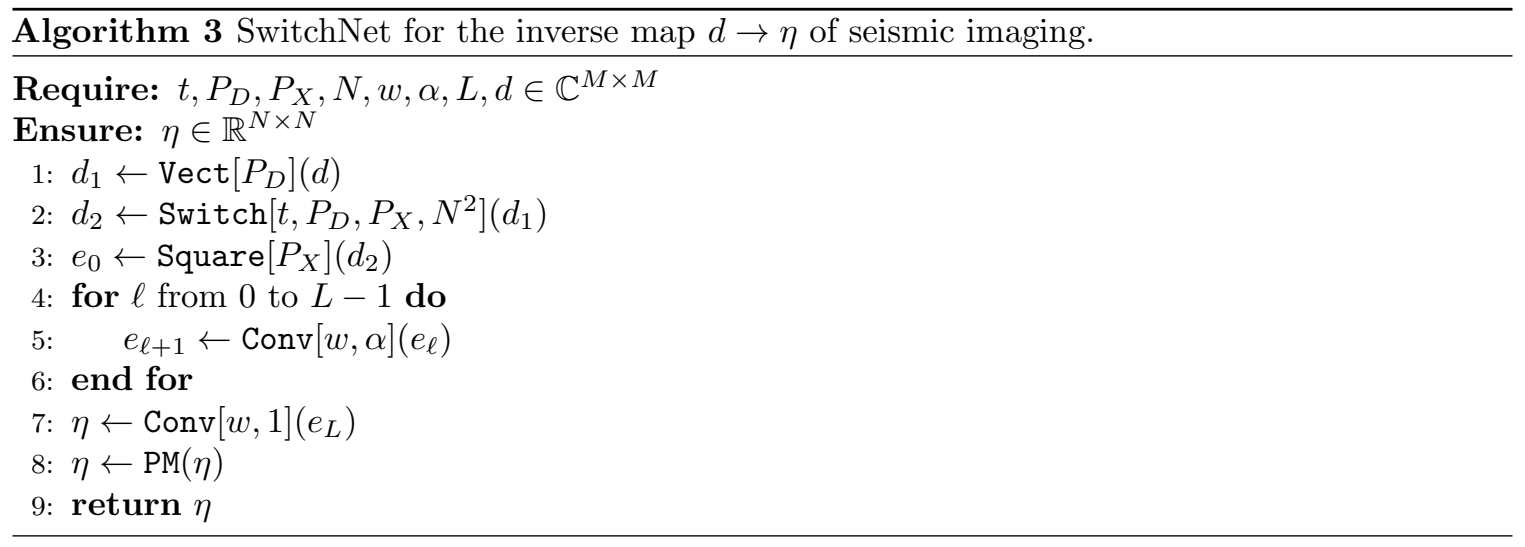

4.3.2. $N N$ for the forward map $\eta \rightarrow d$. As in Section 3.4.3. for the forward map from $\eta \rightarrow d$, we simply reverse the architecture of the NN for the inverse map in Algorithm 3 . For completeness we detail its structure in Algorithm 4. The main difference between Algorithm 2 and Algorithm 4 is again the inclusion of an extra pointwise multiplication layer.

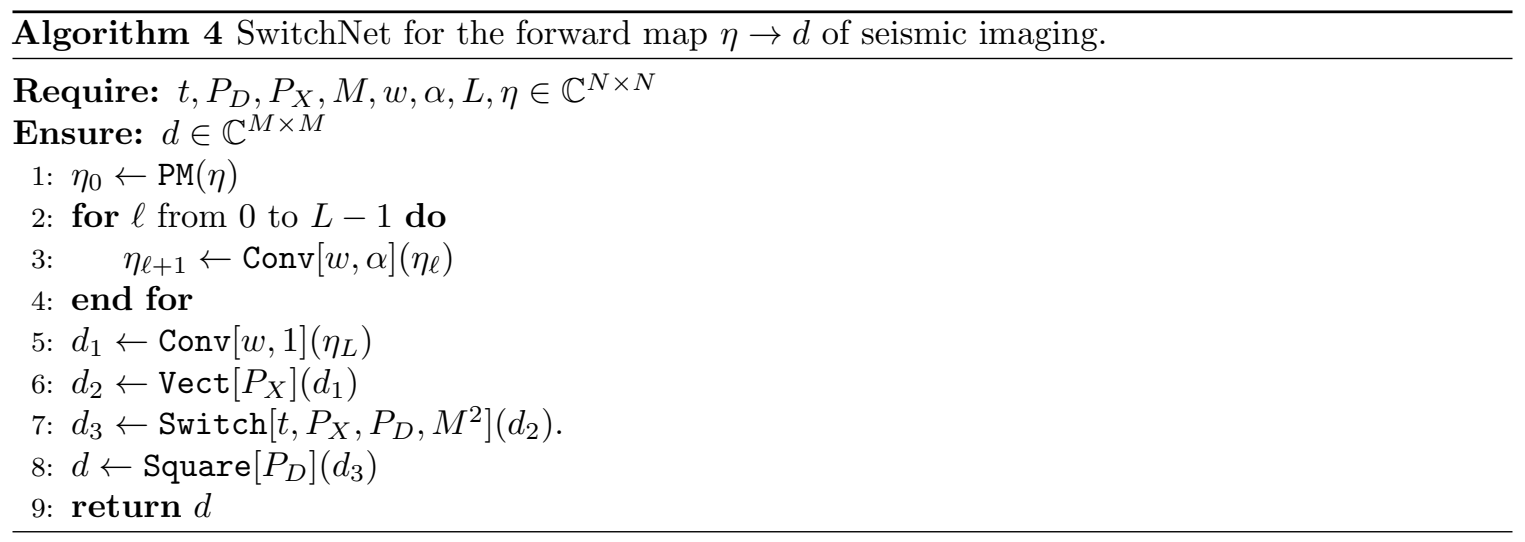

4.4. Numerical results. In the numerical experiments, we set $\Omega=[-0.5,0.5]^{2}$ and discretize it by a $64 \times 64$ Cartesian grid. As mentioned before, the sources $S$ and the receivers $R$ are located on a line near the top surface of $\Omega$, similar to the setting in Fig. 6. This line is discretized uniformly with $M=80$ points. Therefore, the size of $\eta$ and $d$ are $64 \times 64$ and $80 \times 80$, respectively. We assume a Gaussian mixture model for $\eta$ as in (38), where $\beta=0.2, \sigma=0.015$. Unlike before, the centers $\left\{c_{i}\right\}_{i=1}^{n_{s}}$ are kept away from the top surface of $\Omega$ in order to ensure that they are well-separated from the sources and receivers.

In Algorithm 3 and Algorithm 4 the parameters are set to be $t=3, P_{X}=8^{2}, P_{D}=4^{2}, N=64$, $M=80, w=8, \alpha=18$, and $L=3$, resulting NNs with $2900 \mathrm{~K}$ parameters. The procedure of training the NNs is the same as the one used in Section 3.5. Table 2 presents the test errors for this model problem. The predicted and the ground truth $d, \eta$ are visually compared in Figure 7 for one typical test sample. 


\begin{tabular}{ccc}
\hline \hline$n_{s}$ & Forward map & Inverse map \\
\hline \hline 2 & $5.6 \mathrm{e}-02$ & $2.1 \mathrm{e}-02$ \\
3 & $7.7 \mathrm{e}-02$ & $2.2 \mathrm{e}-02$ \\
4 & $8.0 \mathrm{e}-02$ & $5.1 \mathrm{e}-02$
\end{tabular}

TABle 2 Prediction error of SwitchNet for the maps $\eta \rightarrow d$ and $d \rightarrow \eta$ for seismic imaging.

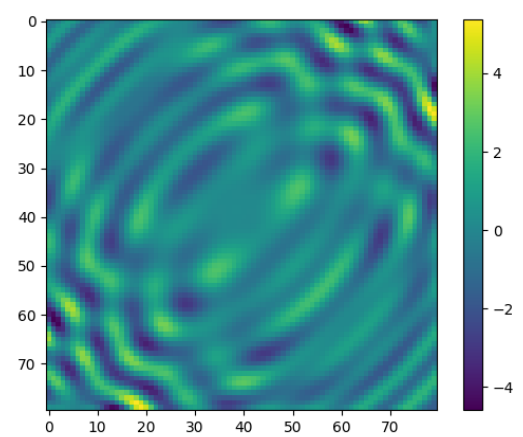

(A) Ground truth scattering pattern $d$.

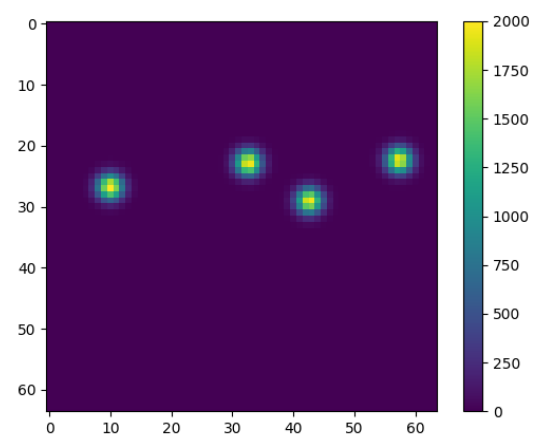

(c) Ground truth scatterers $\eta=$ $\omega^{2}\left(1 / c^{2}-1 / c_{0}^{2}\right)$.

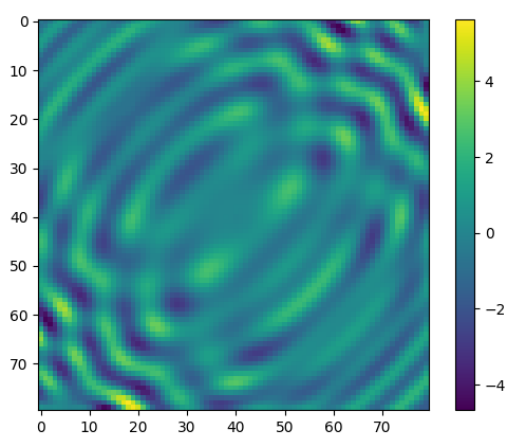

(B) Predicted scattering pattern $d^{\mathrm{NN}}$.

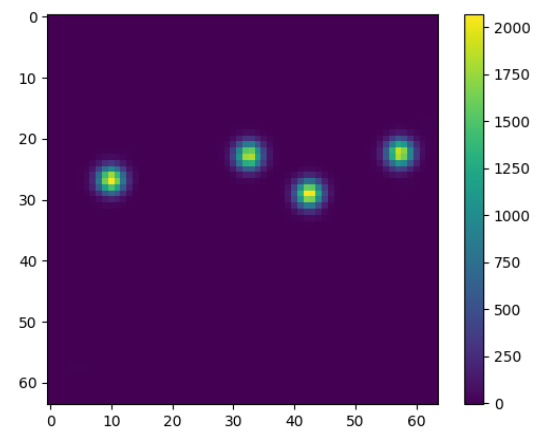

(D) Predicted scatterers $\eta^{\mathrm{NN}}$

FIgURE 7 Results for a typical instance of the seismic imaging setting with $n_{s}=4$. (a) The ground truth scattering pattern. (b) The scattering pattern predicted by SwitchNet with a 7.7e-02 relative error. (c) The ground truth scatterers. (d) The scatterers predicted by SwitchNet with a $6.9 \mathrm{e}-02$ relative error.

\section{Discussion}

In this paper, we introduce a neural network, SwitchNet, for approximating forward and inverse maps arising from the time-harmonic wave equation. For these maps, local information at the input 
has a global impact at the output, therefore they generally require the use of a fully connected NN in order to parameterize them. Based on certain low-rank property that arises in the linearized operators, we are able to replace a fully connected NN with the sparse SwitchNet, thus reducing complexity dramatically. Furthermore, unlike convolutional NNs with local filters, the proposed SwitchNet connects the input layer with the output layer globally. This enables us to represent highly oscillatory wave field resulted from scattering problems, and to solve for the associated inverse problems.

\section{ACKNOWLEDGMENTS}

The work of Y.K. and L.Y. is partially supported by the U.S. Department of Energy, Office of Science, Office of Advanced Scientific Computing Research, Scientific Discovery through Advanced Computing (SciDAC) program and the National Science Foundation under award DMS-1818449. Y.K. thanks Prof. Emmanuel Candès for the partial support from a Math+X postdoctoral fellowship. This work is also supported by the GCP Research Credits Program from Google.

\section{REFERENCES}

[1] J.-P. Berenger. A perfectly matched layer for the absorption of electromagnetic waves. Journal of computational physics, 114(2):185-200, 1994.

[2] E. Candès, L. Demanet, and L. Ying. A fast butterfly algorithm for the computation of Fourier integral operators. Multiscale Modeling \& Simulation, 7(4):1727-1750, 2009.

[3] G. Carleo and M. Troyer. Solving the quantum many-body problem with artificial neural networks. Science, 355(6325):602-606, 2017.

[4] F. Chollet. Keras (2015). URL http://keras. io, 2017.

[5] D. Colton and R. Kress. Inverse acoustic and electromagnetic scattering theory, volume 93 of Applied Mathematical Sciences. Springer, New York, third edition, 2013.

[6] W. E and B. Yu. The deep Ritz method: A deep learning-based numerical algorithm for solving variational problems. Communications in Mathematics and Statistics, 6(1):1-12, 2018.

[7] B. Engquist and H. Zhao. Approximate separability of the green's function of the Helmholtz equation in the high frequency limit. Communications on Pure and Applied Mathematics, 71(11):2220-2274, 2018.

[8] Y. Fan, L. Lin, L. Ying, and L. Zepeda-Núnez. A multiscale neural network based on hierarchical matrices. Arxiv preprint arXiv:1807.01883, 2018.

[9] I. Goodfellow, Y. Bengio, and A. Courville. Deep learning. MIT press, 2016

[10] J. Han, A. Jentzen, and E. Weinan. Solving high-dimensional partial differential equations using deep learning. Proceedings of the National Academy of Sciences, page 201718942, 2018.

[11] J. Han, L. Zhang, R. Car, et al. Deep potential: A general representation of a many-body potential energy surface. Arxiv preprint arXiv:1707.01478, 2017.

[12] G. E. Hinton and R. R. Salakhutdinov. Reducing the dimensionality of data with neural networks. Science, 313(5786):504-507, 2006.

[13] Y. Khoo, J. Lu, and L. Ying. Solving parametric pde problems with artificial neural networks. Arxiv preprint arXiv:1707.03351, 2017.

[14] Y. Khoo, J. Lu, and L. Ying. Solving for high dimensional committor functions using artificial neural networks. Arxiv preprint arXiv:1802.10275, 2018.

[15] D. Kingma and J. Ba. Adam: A method for stochastic optimization. Arxiv preprint arXiv:1412.6980, 2014.

[16] I. E. Lagaris, A. Likas, and D. I. Fotiadis. Artificial neural networks for solving ordinary and partial differential equations. IEEE Transactions on Neural Networks, 9(5):987-1000, 1998.

[17] S. Larsson and V. Thomée. Partial differential equations with numerical methods, volume 45 of Texts in Applied Mathematics. Springer-Verlag, Berlin, 2009. Paperback reprint of the 2003 edition.

[18] Y. LeCun, Y. Bengio, and G. Hinton. Deep learning. Nature, 521(7553):436-444, 2015.

[19] Y. Li, X. Cheng, and J. Lu. Butterfly-net: Optimal function representation based on convolutional neural networks. Arxiv preprint arXiv:1805.07451, 2018.

[20] Y. Li and H. Yang. Interpolative butterfly factorization. SIAM Journal on Scientific Computing, 39(2):A503-A531, 2017.

[21] Y. Li, H. Yang, E. R. Martin, K. L. Ho, and L. Ying. Butterfly factorization. Multiscale Modeling ES Simulation, $13(2): 714-732,2015$. 
[22] S. Liu and G. Trenkler. Hadamard, Khatri-Rao, Kronecker and other matrix products. Int. J. Inf. Syst. Sci, 4(1):160-177, 2008.

[23] Z. Long, Y. Lu, X. Ma, and B. Dong. PDE-net: Learning PDEs from data. Arxiv preprint arXiv:1710.09668, 2017.

[24] T. Mikolov, M. Karafiát, L. Burget, J. Černockỳ, and S. Khudanpur. Recurrent neural network based language model. In Eleventh Annual Conference of the International Speech Communication Association, 2010.

[25] F. Natterer. The mathematics of computerized tomography, volume 32 of Classics in Applied Mathematics. Society for Industrial and Applied Mathematics (SIAM), Philadelphia, PA, 2001. Reprint of the 1986 original.

[26] R. Pascanu, T. Mikolov, and Y. Bengio. On the difficulty of training recurrent neural networks. In International Conference on Machine Learning, pages 1310-1318, 2013.

[27] K. Rudd and S. Ferrari. A constrained integration (CINT) approach to solving partial differential equations using artificial neural networks. Neurocomputing, 155:277-285, 2015.

[28] J. Schmidhuber. Deep learning in neural networks: An overview. Neural networks, 61:85-117, 2015.

[29] L. Ying. Sparse fourier transform via butterfly algorithm. SIAM Journal on Scientific Computing, 31(3):1678-1694, 2009.

Department of Mathematics, Stanford University, Stanford, CA 94305.

E-mail address: ykhoo@stanford.edu

Department of Mathematics and ICME, Stanford University, Stanford, CA 94305. Facebook Ai Research, Menlo Park, CA 94025.

E-mail address: lexing@stanford.edu 